\title{
Ascent, descent and roots of Fredholm operators
}

\author{
by \\ Bertram Yood (University Park, PA)
}

\begin{abstract}
Let $T$ be a Fredholm operator on a Banach space. Say $T$ is rootless if there is no bounded linear operator $S$ and no positive integer $m \geq 2$ such that $S^{m}=T$. Criteria and examples of rootlessness are given. This leads to a study of ascent and descent whether finite or infinite for $T$ with examples having infinite ascent and descent.
\end{abstract}

1. Introduction. Let $X$ be a linear space of sequences, say $X=\ell_{2}$. This paper originated with the realization that the familiar shift operators $T_{1}(x)=\left(0, x, x_{2}, \ldots\right)$ and $T_{2}(x)=\left(x_{2}, x_{3}, \ldots\right)$ are rootless in the sense that there is no bounded linear operator $S$ on $X$ and no positive integer $m \geq 2$ where $S^{m}=T_{k}$. We first sought general criteria for rootlessness and other examples. One such result is that a Fredholm linear operator $T$ on a Banach space is rootless if $T$ has infinite ascent and one-dimensional null space. This study is intimately concerned with the notions of index, ascent and descent for a Fredholm linear operator $T$. The question of when the ascent and descent of $T$ are finite or infinite is of interest. We seek criteria which do not involve the powers $T^{m}$. For example every $T$ with negative index has infinite descent. Every $T$ with finite ascent and descent has index zero. The set of such $T$ is characterized algebraically in Section 3 with no mention of the powers $T^{m}$.

Next let $T$ be any bounded linear operator. In Section 4 we examine the essential resolvent set for $T$, the set of complex $\lambda$ for which $\lambda I-T$ is a Fredholm operator. The subset of such $\lambda$ where $\lambda I-T$ has finite ascent and descent is open. Examples with all possible indices show that the subset where $\lambda I-T$ has infinite ascent and descent can be open and not void.

For early work on ascent and descent see the article of A. E. Taylor [6]. For later results we cite [3], [4] and [5].

2. Rootless operators. Henceforth $X$ will denote an infinite-dimensional Banach space. Let $\mathfrak{B}(X)$ (resp. $\mathfrak{K}(X)$ ) denote the algebra of all bounded linear (resp. compact) operators on $X$ and $\Phi(X)$ be the set of all Fred-

2000 Mathematics Subject Classification: Primary 47A53. 
holm operators in $\mathfrak{B}(X)$. For $T \in \mathfrak{B}(X)$ let $N(T)$ and $R(T)$ denote, respectively, the null space and range space of $T$. Let $\alpha(T)$ be the dimension of $N(T)$ and $\beta(T)$ that of $X / R(T)$. For $T \in \Phi(X)$ the index of $T$, $\operatorname{ind}(T)$, is given by $\operatorname{ind}(T)=\alpha(T)-\beta(T)$. Let $S, T \in \Phi(X)$. Then $S T \in \Phi(X)$ and we have the index formula

$$
\operatorname{ind}(S T)=\operatorname{ind}(S)+\operatorname{ind}(T) .
$$

See for example [1, Th. 3.2.7]. Also $T^{*} \in \Phi\left(X^{*}\right)$ and $\alpha\left(T^{*}\right)=\beta(T), \alpha(T)$ $=\beta\left(T^{*}\right)$ by $[1$, Prop. 1.2.7].

We say that $T \in \Phi(X)$ is rootless if there is no $S \in B(X)$ and no integer $m \geq 2$ such that $S^{m}=T$. If any such $S$ exists then $S \in \Phi(X)$ by [1, p. 9].

Lemma 2.1. Let $T \in \Phi(X)$. There is a positive integer $N$ and nonnegative integers $C_{\mathrm{a}}(T)$ and $C_{\mathrm{d}}(T)$ where $\alpha\left(T^{n+1}\right)-\alpha\left(T^{n}\right)=C_{\mathrm{a}}(T)$ and $\beta\left(T^{n+1}\right)-\beta\left(T^{n}\right)=C_{\mathrm{d}}(T)$ for all $n \geq N$. Also $\operatorname{ind}(T) \leq C_{\mathrm{a}}(T) \leq \alpha(T)$ and $-\operatorname{ind}(T) \leq C_{\mathrm{d}}(T) \leq \beta(T)$ and $\alpha\left(T^{n}\right) \leq n \alpha(T)$ for all $n$.

Proof. For each positive integer $j$ we see, by $(2.1)$, that $\operatorname{ind}\left(T^{j}\right)=$ $j$ ind $(T)$. Hence

$$
\alpha\left(T^{n+1}\right)-\alpha\left(T^{n}\right)-\left[\beta\left(T^{n+1}\right)-\beta\left(T^{n}\right)\right]=\operatorname{ind}(T) .
$$

For $T_{1}, T_{2} \in \Phi(X)$ we have the relation

$$
\alpha\left(T_{1} T_{2}\right)=\alpha\left(T_{2}\right)+\operatorname{dim}\left[T_{2}(X) \cap T_{1}^{-1}(0)\right] .
$$

We use this with $T_{1}=T$ and $T_{2}=T^{n}$ to obtain

$$
\alpha\left(T^{n+1}\right)-\alpha\left(T^{n}\right)=\operatorname{dim}\left[T^{n}(X) \cap T_{1}^{-1}(0)\right] .
$$

Therefore, setting $\Delta_{n}=\alpha\left(T^{n+1}\right)-\alpha\left(T^{n}\right)$, we see by (2.4) that $\Delta_{n+1} \leq \Delta_{n}$. Each $\Delta_{n}$ is a non-negative integer. Hence there is a non-negative integer $C_{\mathrm{a}}(T)$ and a positive integer $N$ such that $\Delta_{n}=C_{\mathrm{a}}(T)$ for all $n \geq N$. Then $\alpha\left(T^{N+r}\right)=\alpha\left(T^{N}\right)+r C_{\mathrm{a}}(T)$ for all positive integers $r$ so that also

$$
\alpha\left(T^{N+r}\right) /(N+r)=\alpha\left(T^{N}\right) /(N+r)+r C_{\mathrm{a}}(T) /(N+r) .
$$

Since $\lim _{r} 1 /(N+r)=0$ and $\lim _{r} r /(N+r)=1$ we see that $\lim \alpha\left(T^{j}\right) / j=$ $C_{\mathrm{a}}(T)$. Next we use (2.3) with $T_{1}=T^{n-1}$ and $T_{2}=T$ to see that $\alpha\left(T^{n}\right) \leq$ $\alpha(T)+\alpha\left(T^{n-1}\right)$ for each positive integer $n$. Thus $\alpha\left(T^{2}\right) \leq 2 \alpha(T)$. We proceed by mathematical induction. Suppose that $\alpha\left(T^{j}\right) \leq j \alpha(T)$ for a positive integer $j$. Then

$$
\alpha\left(T^{j+1}\right) \leq \alpha(T)+\alpha\left(T^{j}\right) \leq(j+1) \alpha(T) .
$$

Therefore $\alpha\left(T^{n}\right) / n \leq \alpha(T)$ for all positive integers $n$. But as $\alpha\left(T^{n}\right) / n \rightarrow$ $C_{\mathrm{a}}(T)$ we have $C_{\mathrm{a}}(T) \leq \alpha(T)$. Also, by $(2.2)$, we see that $\operatorname{ind}(T) \leq C_{\mathrm{a}}(T)$.

Likewise there is a positive integer $m$ such that, for $n \geq m$, $\operatorname{ind}\left(T^{*}\right) \leq$ $\alpha\left(T^{*(n+1)}\right)-\alpha\left(T^{* n}\right) \leq \alpha\left(T^{*}\right)$. Therefore $-\operatorname{ind}(T) \leq C_{\mathrm{d}}(T) \leq \beta(T)$. 
Proposition 2.2. Let $T \in \Phi(X)$. If $T$ has finite ascent (resp. descent) then $\operatorname{ind}(T) \leq 0$ (resp. $\geq 0)$. If $T$ has both finite ascent and descent then $\operatorname{ind}(T)=0$.

Proof. Finite ascent (resp. descent) for $T$ is equivalent to $C_{\mathrm{a}}(T)=0$ (resp. $\left.C_{\mathrm{d}}(T)=0\right)$. We apply Lemma 2.1 .

TheOREM 2.3. Let $T \in \Phi(X)$. If $\operatorname{ind}(T)=0$ then $T$ has finite ascent if and only if it has finite descent. If $\operatorname{ind}(T)>0$ (resp. $<0)$ then $T$ has infinite ascent (resp. descent). If $T$ has finite ascent (resp. descent) and infinite descent (resp. ascent) then $\operatorname{ind}(T)<0$ (resp. $>0)$.

Proof. Suppose ind $(T)=0$. By (2.1) we have $\operatorname{ind}\left(T^{n}\right)=n \operatorname{ind}(T)=0$ for all positive integers $n$.

If $\operatorname{ind}(T)>0$ we must, by Lemma 2.1 , have $C_{\mathrm{a}}(T)>0$, and if $\operatorname{ind}(T)<0$ we have $C_{\mathrm{d}}(T)>0$. Suppose that $T$ has finite ascent and infinite descent. Then $\operatorname{ind}(T) \neq 0$. But we cannot have $\operatorname{ind}(T)>0$ for otherwise $T$ would have infinite ascent.

TheOREM 2.4. Let $T \in \Phi(X)$. Then $\alpha\left(T^{n}\right)=n \alpha(T)$ for all positive integers $n$ if and only if $\alpha(T)=C_{\mathrm{a}}(T)$. If $\alpha(T)=1$ and $T$ has infinite ascent then $\alpha\left(T^{n}\right)=n$ for all $n$.

Proof. Suppose that $\alpha\left(T^{n}\right)=n \alpha(T)$ for all positive integers $n$. As seen in the proof of Lemma 2.1, $\alpha\left(T^{n}\right) / n \rightarrow C_{\mathrm{a}}(T)$. Therefore $C_{\mathrm{a}}(T)=\alpha(T)$.

Conversely, suppose that $C_{\mathrm{a}}(T)=\alpha(T)$. As seen in Lemma 2.1, $\alpha\left(T^{n}\right) \leq n \alpha(T)$ for all positive integers $n$. But $\alpha\left(T^{n+1}\right)-\alpha\left(T^{n}\right) \geq C_{\mathrm{a}}(T)$ for all $n$. From this we see that $\alpha\left(T^{n}\right) \geq n \alpha(T)$ for all $n$.

If $\alpha(T)=1$ then, by Lemma 2.1 , either $C_{\mathrm{a}}(T)=1$ or $C_{\mathrm{a}}(T)=0$. If also $T$ has infinite ascent then $C_{\mathrm{a}}(T)=1$.

Lemma 2.5. Let $T \in \Phi(X)$. For a positive integer $p, C_{\mathrm{a}}\left(T^{p}\right)=p C_{\mathrm{a}}(T)$. Also $C_{\mathrm{a}}(T)-C_{\mathrm{d}}(T)=\operatorname{ind}(T)$.

Proof. We have

$$
\alpha\left(T^{p(n+1)}\right)-\alpha\left(T^{p n}\right)=\sum_{j=1}^{p}\left[\alpha\left(T^{p n+j}\right)-\alpha\left(T^{p n+j-1)}\right] .\right.
$$

By Lemma 2.1 we see that $C_{\mathrm{a}}\left(T^{p}\right)=p C_{\mathrm{a}}(T)$.

By the index formula ind $\left(T^{n}\right)=n \operatorname{ind}(T)$ for all positive integers $n$. For $n$ sufficiently large $C_{\mathrm{a}}(T)=\alpha\left(T^{n}\right)-\alpha\left(T^{n-1}\right)$ and also $C_{\mathrm{d}}(T)=\beta\left(T^{n}\right)-$ $\beta\left(T^{n-1}\right)$. Then $C_{\mathrm{a}}(T)-C_{\mathrm{d}}(T)=\operatorname{ind}\left(T^{n}\right)-\operatorname{ind}\left(T^{n-1}\right)=\operatorname{ind}(T)$.

TheOREM 2.6. Let $T \in \Phi(X)$. Then $T$ is rootless if $\operatorname{ind}(T)= \pm 1$ or if $C_{\mathrm{a}}(T)=1$. 
Proof. Suppose $T=S^{n}$ for $S \in \mathfrak{B}(X)$. Then $\operatorname{ind}(T)=n \operatorname{ind}(S)$ by (2.1) and $C_{\mathrm{a}}(T)=n C_{\mathrm{a}}(S)$ by Lemma 2.5. Therefore $T=S^{n}$ is impossible for $n \geq 2$.

Corollary 2.7. $T \in \Phi(X)$ is rootless if $\alpha(T)=1$ and $T$ has infinite ascent.

Proof. As noted above, we have $C_{\mathrm{a}}(T)=1$.

Theorem 2.8. Let $T \in \Phi(X)$. Suppose $\operatorname{ind}(T) \neq 0$ and $C_{\mathrm{a}}(T)>0$. Then $T$ is rootless if ind $(T)$ and $C_{\mathrm{a}}(T)$ are relatively prime.

Proof. Suppose $T=S^{n}$ for $S \in \mathfrak{B}(X)$. As above $n \operatorname{ind}(S)=\operatorname{ind}(T)$ and $n C_{\mathrm{a}}(S)=C_{\mathrm{a}}(T)$. Therefore we cannot have $n \geq 2$.

We show that there exist $T \in \Phi(X)$ of all possible indices which are rootless. These examples will have infinite ascent and descent. For index zero we examine an operator $V_{1}$ already considered in $[7$, p. 599] and [1, pp. 13, 14]. Here and below $X$ may be taken as a linear space of sequences $x=\left(x_{1}, x_{2}, \ldots\right)$, say $X=\ell_{2}$. We set

$$
V_{1}(x)=\left(0, x_{4}, x_{1}, x_{6}, x_{3}, \ldots\right)
$$

where, after the $x_{j}$ on the right side, the next entries are those with successive odd and even indices interlacing.

For any linear operator $T$ of this sort where the $x_{j}$ on the right have no repetitions and only a finite number of $x_{j}$ fail to appear on the right, $\alpha(T)$ is the number of missing $x_{j}$ 's. There are only a finite number of zeros on the right and $\beta(T)$ is that number of zeros. Here $\alpha\left(V_{1}\right)=\beta\left(V_{1}\right)$ and $\operatorname{ind}\left(V_{1}\right)=0$. It was shown $\left(\left[7\right.\right.$, p. 599] or $[1$, p. 14] $)$ that $V_{1}$ has infinite ascent. By Theorem 2.3 we see that $V_{1}$ has infinite ascent. By the same theorem, $V_{1}$ has infinite descent. Also $V_{1}$ is rootless by Corollary 2.7 .

Let $n$ be a positive integer. Our definition of $V_{n}(x)$ starts off on the right with successive pairs $0, x_{2 j}$ beginning with $0, x_{4}$ and ending with $0, x_{2(n+1)}$. This is then followed by $x_{1}$, and thereafter the next available $x_{j}$ occur, odd and even indices interlacing. In our formal definition of $V_{n}(x), x_{j}$ with $j$ negative is to be read as zero. We set $V_{n}(x)=\left\{y_{j}\right\}$ where $y_{j}=x_{j+2}$ if $j \geq 2$ is even, and $y_{j}=x_{j-2 n}$ if $j \geq 1$ is odd. Specifically,

$$
V_{n}(x)=\left(0, x_{4}, 0, \ldots, 0, x_{2(n+1)}, x_{1}, \ldots\right) .
$$

There are $n$ zeros on the right and every $x_{j}$ appears except $x_{2}$. Thus $\alpha\left(V_{n}\right)=1$ and $\beta\left(V_{n}\right)=n$ so that $\operatorname{ind}\left(V_{n}\right)=1-n$. Then $V_{n}$ has infinite descent by Theorem 2.3 .

Next we consider the powers $V_{n}^{k}$ of $V_{n}$. Each application of $V_{n}$ moves the entries with even index two spaces to the left, those with odd index $2 n$ spaces to the right. For example

$$
V_{n}^{2}(x)=\left(0, x_{6}, 0, \ldots, 0, x_{4 n}, x_{1}, \ldots\right) .
$$


Let $\delta_{j}=\left\{x_{k}\right\}$ where $x_{j}=1$ and $x_{k}=0$ otherwise. Then $V_{n}^{k}\left(\delta_{2 r}\right)=\delta_{2 r-2 k}$. This shows that $V_{n}^{n-1}\left(\delta_{2 n}\right)=\delta_{2} \neq 0$ and $V_{n}^{n}\left(\delta_{2 n}\right)=0$. Therefore $V_{n}$ has infinite ascent and, by Corollary 2.7, it is rootless.

The operators $V_{n}^{*}$ are rootless and have infinite ascent and descent and have positive indices of index $n-1$.

All the examples of rootless operators presented above have infinite ascent and/or descent. There are examples with finite ascent and descent. The simplest example is the matrix

$$
T=\left(\begin{array}{ll}
0 & 1 \\
0 & 0
\end{array}\right)
$$

which is rootless. To see this suppose that $T=S^{n}$ where

$$
S=\left(\begin{array}{ll}
a & b \\
c & d
\end{array}\right)
$$

The null space of $T$ as a linear operator on the vectors $\left(\begin{array}{l}x \\ y\end{array}\right)$ is the set of all $\left(\begin{array}{l}x \\ 0\end{array}\right)$. The null space of $S$ is contained in that of $T$ so is either zero or one-dimensional. It cannot be zero for otherwise $S$ and so $T$ would be invertible. Then $S\left(\begin{array}{l}1 \\ 0\end{array}\right)=\left(\begin{array}{l}0 \\ 0\end{array}\right)$ so that $a=c=0$. Likewise $(0,1) S=(0,0)$ so that $c=d=0$. Then

$$
S=\left(\begin{array}{ll}
0 & b \\
0 & 0
\end{array}\right)
$$

so that $S^{2}=0$. Hence $T$ is rootless.

A study of rootless matrices is under preparation.

3. On finite ascent and descent. Here we examine $\Gamma(X)$, the set of all $T \in \Phi(X)$ which have finite ascent and descent. Given $T \in \Phi(X)$ there exist infinitely many $U \in \Phi(X)$ so that $T U=I+W_{1}$ and $U T=I+W_{2}$ where each $W_{k} \in \mathfrak{K}(X)$. We show that $T \in \Gamma(X)$ if and only if there exists $U \in \Phi(X)$ such that $T U=U T$.

Lemma 3.1. Let $T, U \in \Phi(X)$ be such that $T U=U T$. Then $T U$ has finite ascent (descent) if and only if $T$ and $U$ have finite ascent (descent).

Proof. As $T U=U T$, we have $\alpha\left((T U)^{n}\right)=\alpha\left(T^{n} U^{n}\right)$. But $\alpha\left(T^{n} U^{n}\right)=$ $\alpha\left(U^{n}\right)+\operatorname{dim}\left[R\left(U^{n}\right) \cap N\left(T^{n}\right)\right]$. Then $\alpha\left(U^{n}\right) \leq \alpha\left((T U)^{n}\right) \leq \alpha\left(T^{n}\right)+\alpha\left(U^{n}\right)$. Thus $T U$ has finite ascent if and only if both $T$ and $U$ have finite ascent. As $T^{*} U^{*}=U^{*} T^{*}$ we may apply this result to $T^{*}$ and $U^{*}$ and use [1, Prop. 1.2.7] to have the conclusion on descents.

Proposition 3.2. Let $T \in \Phi(X), V \in \mathfrak{B}(X)$ where $T=V^{n}$. Then $T$ has finite ascent (descent) if and only if $V$ does.

Proof. First of all $V \in \Phi(X)$ by [1, Cor. 1.3.6]. Clearly $V$ has finite ascent (descent) if $T$ does. The converse follows from Lemma 3.1. 
Theorem 3.3. Let $T \in \Phi(X)$. The following are equivalent:

(a) $T \in \Gamma(X)$.

(b) There exist $U \in \mathfrak{B}(X), W \in \mathfrak{K}(X)$ such that $U T=T U=I+W$.

(c) There exists $U \in \Phi(X)$ such that $U T=T U$ and $U T \in \Gamma(X)$.

Proof. Assume (a). By [1, Th. 1.4.5] we can write $T=V_{1}+V_{2}$ where $V_{1} \in \Phi(X)$ is an isomorphism of $X$ onto $X, V_{2} \in \mathfrak{K}(X)$ and $V_{1} V_{2}=V_{2} V_{1}$. Clearly $V_{1}$ and hence $V_{1}^{-1}$ permutes with $T$. We have $V_{1}^{-1} T=I+V_{1}^{-1} V_{2}$ and $T V_{1}^{-1}=I+V_{2} V_{1}^{-1}$ with $V_{1}^{-1} V_{2}=V_{2} V_{1}^{-1} \in \mathfrak{K}(X)$.

Assume (b). We have $U \in \Phi(X)$ by [1, Cor. 1.3.6] so that (b) implies (c). That (c) implies (a) follows from Lemma 3.1.

Corollary 3.4. Let $T \in \Phi(X)$. Suppose that $U T=T U=I+W$ as in (b) of Theorem 3.3. Let $S \in \mathfrak{B}(X)$ permute with $T$ and $U$. Then there is $\varepsilon>0$ so that $T+\lambda S \in \Gamma(X)$ for all complex $\lambda,|\lambda|<\varepsilon$.

Proof. As $W=U T-I$ and $U T=T U$ we see that $U W=W U$. Also $U(T+\lambda S)=(T+\lambda S) U$ for all complex $\lambda$. For some $\varepsilon_{1}>0, T+\lambda S \in \Phi(X)$ for all $|\lambda|<\varepsilon_{1}$, as $\Phi(X)$ is an open set [1, Th. 4.4.1]. We have $U(T+\lambda S)=$ $I+\lambda U S+W$. As $S$ permutes with $U$ and $T$ it follows that $S W=W S$. Thus $(I+\lambda U S) W=W(I+\lambda U S)$. There exists $\varepsilon_{2}>0$ so that $I+\lambda U S$ is an isomorphism of $X$ onto $X$ for $|\lambda|<\varepsilon_{2}$. Let $|\lambda|<\min \left(\varepsilon_{1}, \varepsilon_{2}\right)$. By [1, Th. 1.4.5], we have $I+\lambda U S+W \in \Gamma(X)$. Then $T+\lambda S \in \Gamma(X)$ by Theorem 3.3.

4. On the essential resolvent set. As in the standard text [2, p. 358], by the essential spectrum of $T \in \mathfrak{B}(X)$ we mean the set of complex $\lambda$ for which $\lambda I-T \notin \Phi(X)$. By the essential resolvent set $\mathfrak{E}(T)$ of $T$ we mean its complement, the set of $\lambda$ for which $\lambda I-T \in \Phi(X)$. The set $\mathfrak{E}(T)$ is open in $\mathfrak{B}(X)$ by $[1$, Th. 4.4.1].

TheOREm 4.1. The set of complex $\lambda \in \mathfrak{E}(T)$ for which $\lambda I-T$ has finite ascent and descent is open.

Proof. The choice $S=I$ in Corollary 3.4 shows that if $\lambda_{0} I-T \in \Gamma(X)$ then, for some $\varepsilon>0$, so does $\lambda I-T$ if $\left|\lambda-\lambda_{0}\right|<\varepsilon$.

Let $T$ be a fixed element of $\mathfrak{B}(X)$. We adopt the following notation. Let $\mathfrak{F}_{\mathrm{a}}$ (resp. $\mathfrak{F}_{\mathrm{d}}$ ) be the set of $\lambda \in \mathfrak{E}(T)$ for which $\lambda I-T$ has finite ascent (resp. descent). Let $\mathfrak{I}_{\mathrm{a}}$ (resp. $\mathfrak{I}_{\mathrm{d}}$ ) be the set of $\lambda \in \mathfrak{E}(T)$ for which $\lambda I-T$ has infinite ascent (resp. descent).

THEOREM 4.2. Suppose that $\mathfrak{I}_{\mathrm{a}} \cap \mathfrak{I}_{\mathrm{d}}$ is relatively closed as a subset of $\mathfrak{E}(T)$. Then $\mathfrak{F}_{\mathrm{a}}, \mathfrak{F}_{\mathrm{d}}, \mathfrak{F}_{\mathrm{a}} \cap \mathfrak{I}_{\mathrm{d}}$ and $\mathfrak{F}_{\mathrm{d}} \cap \mathfrak{I}_{\mathrm{a}}$ are open subsets of $\mathfrak{B}(X)$. 
Proof. Let $\lambda_{0} \in \mathfrak{F}_{\mathrm{a}} \cap \mathfrak{I}_{\mathrm{d}}$. By hypothesis there exists $\varepsilon_{1}>0$ so that $\lambda \in \mathfrak{F}_{\mathrm{a}} \cup \mathfrak{F}_{\mathrm{d}}$ for $\left|\lambda-\lambda_{0}\right|<\varepsilon_{1}$. By Theorem 2.3, ind $\left(\lambda_{0} I-T\right)<0$. Then, by [1, Th. 4.4.1], there is $\varepsilon_{2}>0$ so that ind $(\lambda I-T)<0$ if $\left|\lambda-\lambda_{0}\right|<\varepsilon_{2}$. By Theorem 2.3, $\lambda \in \mathfrak{I}_{\mathrm{d}}$ for these $\lambda$. Therefore $\lambda \in \mathfrak{F}_{\mathrm{a}} \cap \mathfrak{I}_{\mathrm{d}}$ if $\left|\lambda-\lambda_{0}\right|<$ $\min \left(\varepsilon_{1}, \varepsilon_{2}\right)$. Now $\mathfrak{F}_{\mathrm{a}} \cap \mathfrak{F}_{\mathrm{d}}$ is open by Theorem 4.4.1 of [1]. This also follows by Corollary 3.4. Therefore $\mathfrak{F}_{\mathrm{a}}$ is open. Likewise $\mathfrak{F}_{\mathrm{d}}$ and $\mathfrak{F}_{\mathrm{d}} \cap \mathfrak{I}_{\mathrm{a}}$ are open.

We show, by example, that we can have $\mathfrak{I}_{\mathrm{a}} \cap \mathfrak{I}_{\mathrm{d}}$ non-void as well as being open and closed in $\mathfrak{E}(T)$.

Let $X=\ell_{2}$. For $x=\left(\xi_{1}, \xi_{2}, \ldots\right)$ in $X$ we treat the operator $V_{1}(x)=$ $\left(0, \xi_{4}, \xi_{1}, \xi_{6}, \xi_{3}, \ldots\right)$ of index zero discussed above. As $\left\|V_{1}^{n}\right\|=1$ for all positive integers $n$, its spectrum is contained in the unit disc of the complex plane. For each $\lambda$ with $|\lambda|<1, N\left(\lambda I-V_{1}\right)$ is the set of scalar multiples of

$$
z=\left(0,1,0, \lambda, 0, \lambda^{2}, \ldots, 0, \lambda^{n}, \ldots\right) .
$$

Hence $\operatorname{sp}\left(V_{1}\right)$ is the entire unit disc. For $|\lambda|=1$ we show that $R\left(\lambda I-V_{1}\right)$ is not closed. First note that $\lambda I-V_{1}$ is one-to-one. Then $R\left(\lambda I-V_{1}\right) \neq X$ as $\lambda \notin \operatorname{sp}\left(V_{1}\right)$. Suppose $R\left(\lambda I-V_{1}\right) \neq X$ is closed. Then, by [1, Th. 2.5.6], there is a neighborhood of $\lambda$ in which $\mu I-V_{1}$ has closed range $\neq X$. This is impossible as the neighborhood contains $\mu,|\mu|>1$. In particular $\lambda I-V_{1} \notin$ $\Phi(X)$ if $|\lambda|=1$.

We consider the adjoint $V_{1}^{*}$ of $V_{1}$,

$$
V_{1}^{*}(x)=\left(\xi_{3}, 0, \xi_{5}, \xi_{2}, \xi_{7}, \xi_{4}, \ldots\right),
$$

where $\xi_{j}$ with odd and even $j$ interlace. We find

$$
V_{1}^{*} V_{1}(x)=\left(\xi_{1}, 0, \xi_{3}, \xi_{4}, \xi_{5}, \ldots\right), \quad V_{1} V_{1}^{*}(x)=\left(0, \xi_{2}, \xi_{3}, \xi_{4}, \xi_{5}, \ldots\right) .
$$

Thus each of $V_{1}^{*} V_{1}$ and $V_{1} V_{1}^{*}$ is of the form $I+W$ where $W \in \mathfrak{K}(X)$. Let $\pi$ be the canonical homomorphism of $\mathfrak{B}(X)$ onto the $C^{*}$-algebra $\mathfrak{B}(X) / \mathfrak{K}(X)$. Then $\pi\left(V_{1}\right)$ is a unitary element in that $C^{*}$-algebra. Therefore the essential spectrum of $V_{1}$ is contained in $\{\lambda:|\lambda|=1\}$. As $R\left(\lambda I-V_{1}\right)$ is not closed for these values of $\lambda$ we see that the unit circle is the essential spectrum. Therefore $\lambda I-V_{1} \in \Phi(X)$ if $|\lambda|<1$, and by [1, Th. 4.4.1], ind $\left(\lambda I-V_{1}\right)=0$.

In view of Theorem 2.3, to show that $\lambda I-V_{1}$ has infinite ascent and descent, it is enough to show that $z$ is in the range of every $\left(\lambda I-V_{1}\right)^{n}$. Let $Q$ denote the set of all $x=\left(\xi_{1}, \xi_{2}, \ldots\right)$ where $\xi_{j}=0$ for all $j$ odd. Note that $z \in Q$. Let $x=\left(0, a_{2}, 0, a_{4}, \ldots\right)$ be a general element of $Q$. Then

$$
\left(\lambda I-V_{1}\right)(x)=\left(0, \lambda a_{2}-a_{4}, 0, \lambda a_{4}-a_{6}, \ldots\right) .
$$

Now $z \in Q$ has $a_{2 n}=\lambda^{n-1}$. Then

$$
\left(\lambda I-V_{1}\right)\left(w_{1}\right)=z \quad \text { where } \quad w_{1} \in Q \text { with } a_{2 n}=-(n-1) \lambda^{n-2} .
$$

Likewise $\left(\lambda I-V_{1}\right)\left(w_{2}\right)=w_{1}$ where $w_{2} \in Q$ with $a_{2 n}=(n-1)(n-2) \lambda^{n-3} / 2$ !. 
More generally we define $w_{k} \in Q$ by

$$
a_{2 n}=(-1)^{k}(n-1)(n-2) \ldots(n-k) \lambda^{n-k-1} / k ! .
$$

Then $\left(\lambda I-V_{1}\right)\left(w_{k}\right)=w_{k-1}$.

Hence $\lambda I-V_{1}$ has infinite ascent and infinite descent by Theorem 2.3. Thus the set $\mathfrak{I}_{\mathrm{a}} \cap \mathfrak{I}_{\mathrm{d}}$ for $V_{1}$ is the open unit disc. Now $\mathfrak{E}\left(V_{1}\right)$ is the complex plane with the unit circle deleted. Thus $\mathfrak{I}_{\mathrm{a}} \cap \mathfrak{I}_{\mathrm{d}}$ is open and closed as a set in $\mathfrak{E}\left(V_{1}\right)$ as well as being non-void.

Examples with the same conclusion can be found with any preassigned index. Consider the operators $V_{n}$ and $V_{n}^{*}$ introduced in Section 2. For these, $\mathfrak{I}_{\mathrm{a}} \cap \mathfrak{I}_{\mathrm{d}}$ have the properties shown for $V_{1}$ while $\lambda I-V_{n}$ has index $1-n$, and $\lambda I-V_{n}^{*}$ has index $n-1$ for all $\lambda,|\lambda|<1$. We omit the details which parallel those for $V_{1}$.

\section{References}

[1] S. R. Caradus, W. Pfaffenberger and B. Yood, Calkin Algebras and Algebras of Operators on Banach Spaces, Dekker, New York, 1974.

[2] J. B. Conway, A Course in Functional Analysis, 2nd ed., Springer, New York, 1990.

[3] S. Grabiner, Ascent, descent and compact perturbations, Proc. Amer. Math. Soc. 71 (1978), 79-80.

[4] M. A. Kaashoek and D. C. Lay, Ascent, descent, and commuting permutations, Trans. Amer. Math. Soc. 169 (1972), 35-47.

[5] V. Rakočević, Semi-Fredholm operators with finite ascent or descent and perturbations, Proc. Amer. Math. Soc. 123 (1995), 3823-3825.

[6] A. E. Taylor, Theorems on ascent, descent, nullity and defect of linear operators, Math. Ann. 163 (1966), 18-49.

[7] B. Yood, Properties of linear transformations preserved under addition of a completely continuous transformation, Duke Math. J. 18 (1951), 599-612.

Department of Mathematics

Pennsylvania State University

University Park, PA 16802, U.S.A.

Received October 17, 2000

Revised version April 10, 2003 